\title{
Inside-Out Infall Formation of Disk Galaxies: Do Predictions Differ from Models without Size Evolution?
}

\author{
Rychard Bouwens \\ Department of Physics,, University of California, Berkeley, CA 94720 \\ Laura Cayón \\ Instituto de Física de Cantabria, Universidad de Cantabria, Spain \\ $\&$ \\ Joseph Silk \\ Departments of Astronomy and Physics, and Center for Particle Astrophysics, University \\ of California, Berkeley, CA 94720
}

\begin{abstract}
We develop an idealized inside-out formation model for disk galaxies to include a realistic mix of galaxy types and luminosities that provides a fair match to the traditional observables. The predictions of our infall models are compared against identical models with no-size evolution by generating fully realistic simulations of the HDF, from which we recover the angular size distributions. We find that our infall models produce nearly identical angular size distributions to those of our no-size evolution models in the case of a $\Omega=0$ geometry but produce slightly smaller sizes in the case of a $\Omega=1$ geometry, a difference we associate with the fact that there is a different amount of cosmic time in our two models for evolving to relatively low redshifts $(z \approx 1-2)$. Our infall models also predict a slightly smaller $(11 \%-29 \%)$ number of large (disk scale lengths $>4 h_{50}^{-1} \mathrm{kpc}$ ) galaxies at $z \approx 0.7$ for the CFRS as well as different increases in the central surface brightness of the disks for early-type spirals, the infall model predicting an increase by 1.2 magnitudes out to $z \approx 2(\Omega=0), 1(\Omega=1)$, while our no-size evolution models predict an increase of only 0.5 magnitude. This result suggests that infall models could be important for explaining the 1.2-1.6 magnitude increase in surface brightness reported by Schade et al. (1995, 1996a, 1996b).
\end{abstract}

Subject headings: Galaxy Evolution - Galaxy Scale-lengths 


\section{Introduction}

Infall formation of disk galaxies predicts an inside-out formation process that produces galaxy sizes smaller than expected in models without intrinsic size evolution (Cayón, Silk \& Charlot 1996). Prantzos \& Aubert (1995) present a detailed comparison of several star formation models in an effort to explain the observed properties of the Galactic disk. The favoured model is one in which the star formation rate (SFR) is proportional to the gas surface density and to the differential rotation rate and in which gas evolution is driven by infall of unenriched gas during a certain period of time in the life of the galaxy (Wang \& Silk 1994). In a first attempt to test infall formation of field disk galaxies, Cayón et al. (1996) noted that the observed trend toward small sizes at faint magnitudes and high redshifts is clearly reproduced by these models.

In this Letter, we extend the development of the idealized infall formation model for disk galaxies by incorporating a range of different galaxy types and luminosities given by a slightly refined version of the Pozzetti, Bruzual, \& Zamorani (1996) pure luminosity evolution (PLE) model. We aim to determine the extent to which these models differ from models with no-size evolution in the prediction of the angular size distributions, the number of large-intermediate redshift disks, and the evolution in surface brightnesses observed in disk galaxies at higher redshifts (Schade et al. 1995, 1996a, 1996b; Simard \& Prichet 1997). All calculations are performed with $H_{o}=50 \mathrm{~km} / \mathrm{sec} / \mathrm{Mpc}$ and expressed in Vega magnitudes unless otherwise noted.

\section{Description of Model}

Infall formation models have been favored by studies of the disk of our own Galaxy (Ferrini et al. 1994; Dopita \& Ryder 1994; see Prantzos \& Aubert 1995 for a detailed comparative analysis) and nearby galaxies (Ryder \& Dopita 1994). A model for disk galaxies based on infall formation was presented in Cayón, Silk \& Charlot (1996). Only a brief description is included here. A phenomenological model satisfying the observational requirements is one with infall in which the SFR depends on radius and time following a Schmidt-type law

$$
S F R(r, t)=(1-R)^{-1} \frac{\Sigma_{g}(r, t)}{\tau_{g}(r)} \quad \mathrm{M}_{\odot} \mathrm{pc}^{-2} \mathrm{Gyr}^{-1},
$$

where $R \approx 0.32$ is the returned fraction of mass that was formed into stars, $\Sigma_{g}(r, t)$ is the gas surface density at radius $r$ and age $t$, and $\tau_{g}(r)=\left[0.3(1-R)\left(r_{\odot} / r\right)\right]^{-1}$ Gyr is the gas consumption timescale $\left(r_{\odot}=8.5 \mathrm{kpc}\right)$. Normalizing the infall rate density of metal-free gas to the total surface density $\Sigma_{t o t}$ of stars plus gas observed at age $T$ Gyr (corresponding to 
$z \sim 0$ ) and using the instantaneous recycling approximation (IRA), the evolution of the gas surface density is given by

$$
\Sigma_{g}(r, t)=\Sigma_{t o t}(r, T) \frac{\exp \left[-t / \tau_{g}(r)\right]-\exp \left[-t / \tau_{f}(r)\right]}{\left\{1-\left[\tau_{f}(r) / \tau_{g}(r)\right]\right\}\left\{1-\exp \left[-T / \tau_{f}(r)\right]\right\}},
$$

where $\tau_{f}$ is the infall time scale. As in our previous work, for galaxies with Milky-way luminosities, i.e., $M_{b_{j}} \approx-20$, we take $\Sigma_{t o t}$ to equal the sum of the observed gas and the stellar surface density necessary to yield the assumed $(z \approx 0)$ surface brightness profile. For galaxies of different luminosities, however, we take the star formation profile to be equal to the star formation profile for Milky-Way galaxies, but stretched by an appropriate factor in radius to produce the luminosity in question.

Closely following the Pozzetti et al. (1996) PLE model, we develop a PLE model in terms of the above formalism for both an open and a flat universe, where we use three different morphological types: ellipticals, early-type spirals, and late-type spirals. To improve the agreement of our model with the number counts observed in the HDF for an open geometry, we increase the normalization and steepen the slope of our luminosity function slightly over the values given by Pozzetti et al. (1996). With these changes, we find that our models fit the number counts, redshift distributions, and luminosity functions as well as does the Pozzetti et al. (1996) model.

We tune both the age $T$ and the gas infall time scale $\tau_{f}$ to match the $z=0$ colours and to provide a rough match with the star formation histories given for the Sabc and Sdm morphological types specified in the Pozzetti et al. (1996) PLE model with exponentially decreasing star formation. We take late-type spirals to have pure exponential profiles, and early-type spirals to have an exponential bulge added to this profile with a scale length that is 0.086 that of the disk (Courteau, de Jong, \& Broeils 1996) with bulge-to-total ratios similar to those given in King \& Ellis (1985). At redshift zero, we assume that our disks follow Freeman's law, i.e. $\mu_{b j}=21.65 \mathrm{mag} / \operatorname{arcsec}^{2}$ (Freeman 1970). For galaxies with $M_{b j}>-18$, however, we take the entire galactic population to be composed of late-type exponential disks with $\mu_{b j}=22.5$ to account for the observed lack of early-type galaxies at low luminosities (Binggeli, Sandage, \& Tammann 1988) and their lower surface brightnesses (McGaugh \& de Blok 1997). For ellipticals, we adopt the size-luminosity relationship given by Binggeli, Sandage, \& Tarenghi (1984). To perform the calculations for the described model, we break up our disk galaxies into approximately 30 annuli, on which we perform the spectral synthesis for each using a recent version of the Bruzual \& Charlot tables (Leitherer et al. 1997). We summarize our choice of parameters in Table 1.

We compare this model, hereinafter referred to as the "Infall" model, with a nearly identical "No Infall" model, in which there is no evolution in the scale length on which star 
formation occurs, even though the star formation history for each morphological type in this model is the same as for the "Infall" model. Furthermore, we ensure that the surface brightnesses in the $b j$ band for both models are exactly the same at $z=0$.

\section{Evolution in Central Surface Brightness and Size}

For our first set of comparisons, we look at the extent to which the "Infall" models differ from "No Infall" models in terms of the evolution of different morphological types in the $\mu_{o}-r_{h l}$ plane. Because of the presence of the bulge in the center of the early-type spirals, we take the central surface brightness $\mu_{o}$ to equal the surface brightness extrapolated assuming an exponential disk profile from the surface brightnesses at $\approx 0.5$ and $\approx 2.0$ disk scale lengths-a procedure that is intended to be an approximate estimate of the central surface brightnesses that would be determined through the multicomponent fits (Schade et al. 1996a). Values in this plane are presented in Figure 1 for the models considered, from redshifts corresponding to an age of 1 Gyr down to $z \sim 0$. The values for $L^{*}$ Sab-Sbc and Scd-Sdm galaxies for the "Infall" model are shown as solid lines in panels (a) and (b), together with the predictions one magnitude above and below (shaded areas). Brightening of approximately 1.2 magnitudes occurs for early types, from $z=0$ to $z \sim 2(\Omega=0.0), 1(\Omega=1.0)$, the rate of brightening being slower in the more open geometry due to the longer time between the formation of the galaxy and $z \approx 1$. These values would be in agreement with recent observations (Schade et al. 1995, 1996a, 1996b) if early-types are dominating the samples. The faster the evolution in the central parts of the disk (larger $T / \tau_{f}$ corresponding to earlier type disks) the larger is the amount of brightening observed. By contrast, our "No Infall" models, presented in panels (c) and (d) of Figure 1, show no such differential evolution, so consequently the early-type galaxies in these models produce only $\sim 0.5$ magnitude compared to the $\sim 1.2$ magnitude of brightening observed in the "Infall" models. These results suggest that inside-out formation scenarios, such as those we sketch in this paper, would not only make a difference in the observations relative to scenarios with no-size evolution, but they also could play an important role in the apparent surface brightness evolution observed to $z \sim 1$.

As is clear from Figure 1, galaxies in our "Infall" models evolve not only in surface brightness but in size relative to "No Infall" models. In light of the present compilation of size information for the CFRS (Lilly et al. 1997), we make a simple estimate for the expected decrease in number of large galaxies predicted in the CFRS at intermediate redshift on the basis of our "Infall" models relative to our "No Infall" models. We find that, for the CFRS selection criteria $\left(17.5<I_{A B}<22.5\right)$, there are 807 (852) large (disk scale 
lengths $>4 \mathrm{kpc})$ galaxies per redshift interval per degree ${ }^{2}$ at $z \approx 0.7$ for our $\Omega=0(\Omega=1)$ infall models compared to 719 (609) for our $\Omega=0(\Omega=1)$ "No Infall" models, a decrease of $11 \%(\Omega=0)$ to $29 \%(\Omega=1)$.

Finally, we examine the differences in the angular size distributions produced by the models in the context of the HDF, the deepest and highest resolution optical observation to date. To this end, we generate detailed images with areas equal to roughly 3.8 times the area of the three WF chips and with properties which closely match those observed in the F814W HDF images. In our simulations, we place galaxies with random orientation angles and no extintion on the mock images assuming no correlation, add Poissonian noise, smooth over a Gaussian kernel with a 0.06-arcsec sigma, and then degrade the image by adding some noise. After adding this noise, we smooth the images again over a Gaussian kernel with a 0.02-arcsec sigma to reproduce approximately the drizzled noise properties of the images (Williams et al. 1996). We use SExtractor 1.2b5 (Bertin \& Arnouts 1996) to make a catalogue and to determine both the magnitude (SExtractor's MAG_BEST) and the half-light radiif of the objects in the F814W images for each of our models as well as the F814W images (version 2) of the three WF chips (1634 objects total). For a detection, we require an object, after smoothing with the PSF, to be composed of at least 10 contiguous pixels at $2 \sigma$ above the background.

As was our goal, in Figure 2 we demonstrate that the $\Omega=0$ models provide fair fits to the HDF number counts, decomposed into three different bins of half-light radii (though we are $20-40 \%$ low in the faintest magnitudes bins). We then compare the angular size distributions recovered from the simulations for our "Infall" models with those recovered from both our "No Infall" models and the HDF in Figure 3, where we decompose our angular size distribution into its constituent morphological types. We find that while both our $\Omega=0$ models are in fair agreement with the angular size distributions found in the HDF, they also appear to be in fair agreement with each other, even for the early-type galaxies which evolve so very differently in the two models (Figure 1). For our $\Omega=1$ models, however, there is a clear difference between the angular size distributions for the "Infall" and "No Infall" models, particularly among the early-type spirals.

To understand this difference, it is important to note that the profiles of galaxies in our "Infall" models tend to approach those in our "No Infall" models over time, and so the convergence begins in the center and grows outward. Fundamentally, both our $\Omega=0$ models produce similar distributions of recovered half-light radii because at any redshift

\footnotetext{
${ }^{1}$ We take the half-light radius to equal the radius of the aperture which contains half the light as determined by SExtractor's best estimate of its total (MAG_BEST).
} 
only those central parts of the galaxy for which the profiles in the two models have started to converge can be seen, these radii being small at high redshifts and large at low redshifts as a result of $(1+z)^{4}$ cosmological dimming. Galaxies in our $\Omega=1$ models, however, have less time to evolve to relatively low redshifts where cosmological dimming is less significant (note that in Figure 1 the redshift corresponding to the peak in surface brightness is lower for our $\Omega=1$ models); therefore, the apparent differences between the profiles produced by the two models can be more readily observed. In Figure 3, we plot the distribution of true half-light radii (calculated from our code which placed each galaxy on our simulated images) for the two $\Omega=0$ models to illustrate the reality of the actual but relatively unobservable differences between these two models.

In summary, we have extended the idealized infall model for disk formation to include a realistic mix of galaxy types and luminosities which provide a rough match to the number counts, redshift distributions, and angular size distributions in the HDF. We compare the predictions of these models against identical models with no-size evolution to see how our infall models would affect the observations. For these infall models, we find that the number of large disks (disk scale lengths $>4 \mathrm{kpc}$ ) is 11\%-29\% smaller than for our no-size evolution models at $z \approx 0.7$ for the CFRS. We also find increases in the central surface brightness of disk galaxies of about $1.2 \mathrm{mag} / \operatorname{arcsec}^{2}$ out to $z \approx 1(\Omega=1), 2(\Omega=0)$ for early-type spirals, very different from the $0.5 \mathrm{mag} / \operatorname{arcsec}^{2}$ increase found for models with no-size evolution. Our finding strongly suggests that inside-out formation scenarios for disk galaxies should have observable consequences at high $z$ and could be important for understanding the observed increase in surface brightness of disks out to $z \approx 1$.

For our $\Omega=0$ models, we find no significant differences between the recoverable angular size distributions predicted on the basis of our infall models and those predicted on the basis of our models with no-size evolution. We suspect that significant differences between these models would have been obtained if the early-type galaxies had been allowed to begin forming at lower redshifts, as we found in the case of our $\Omega=1$ models. However, in this case, there would have not been as many galaxies at high redshifts and our models would have underpredicted the number of galaxies at faint magnitudes. We therefore suspect that for any simple single-formation-epoch PLE models which fit the number counts, infall and no-size evolution models will tend to produce very similar angular size distributions given the current state of observations. Therefore, if our infall models are correct, the fact that other workers (Ferguson \& Babul 1997) have found angular size distributions with no-size evolution PLE models which tend to agree with the HDF observations is not very surprising in light of the present result.

We would like acknowledge Ian Smail for his suggested procedure for the determination 
of half-light radii. We would like to thank Emmanuel Bertin for providing us with SExtractor and Stephane Charlot for making the Bruzual/Charlot spectral synthesis curves available. We acknowledge useful conversations with Steve Zepf and Harry Ferguson. L.C. thanks both the Center for Particle Astrophysics and Berkeley Astronomy Department for their hospitality during her stay in Berkeley. R.J.B. gratefully acknowledges support from an NSF graduate fellowship. This research has also been supported in part by grants from NASA and NSF.

\section{REFERENCES}

Bertin, E., \& Arnouts, S. 1996, A\&AS, 117, 393.

Binggeli, B., Sandage, A., \& Tarenghi, M. 1984, ApJ, 89, 64.

Binggeli, B., Sandage, A., \& Tammann, G.A. 1988, ARA\&A, 26, 509.

Cayón, L., Silk, J. \& Charlot, S. 1996, ApJ, 467, L53.

Courteau, S., de Jong, R.S. \& Broeils, A.H. 1996, ApJ, 457, L73.

Dopita, M. \& Ryder, S. 1994, ApJ, 430, 163.

Ferrini, F., Molla, M., Pardi, M., \& Diaz, A. 1994, ApJ, 427, 745.

King, C.R., Ellis, R.S. 1985, ApJ, 288, 456.

Ferguson, H.C., \& Babul, A. 1997, in preparation.

Freeman, K.C. 1970, ApJ, 160, 811.

Leitherer, C., et al. 1996, 108, 996.

Lilly, S.J., et al. 1997, in preparation.

McGaugh, S.S., \& de Blok, W.J.G. 1997, ApJ, 481, 689.

Pozzetti, L., Bruzual, G. \& Zamorani, G. 1996, MNRAS, 281, 953.

Prantzos, N. \& Aubert, O. 1995, A\&A, 302, 69.

Ryder, S. \& Dopita, M. 1994, ApJ, 430, 142.

Simard, L., \& Pritchet, C.J. 1997, astro-ph/9606006.

Schade, D., Lilly, S.J., Crampton, D., Hammer, F., Le Fevre, O. \& Tresse, L. 1995, ApJ, 451, L1.

Schade, D., Lilly, S.J., Le Fevre, O., Hammer, F. \& Crampton, D. 1996a, ApJ, 464, 79.

Schade, D., Carlberg, R.G., Yee, H.K.C., López-Cruz, O. \& Ellingson, E. 1996b, ApJ, 465, L103. 
Wang, B. Q. \& Silk, J. 1994, ApJ, 427, 759.

Williams, R.E., et al. 1996, AJ, 112, 1335. 


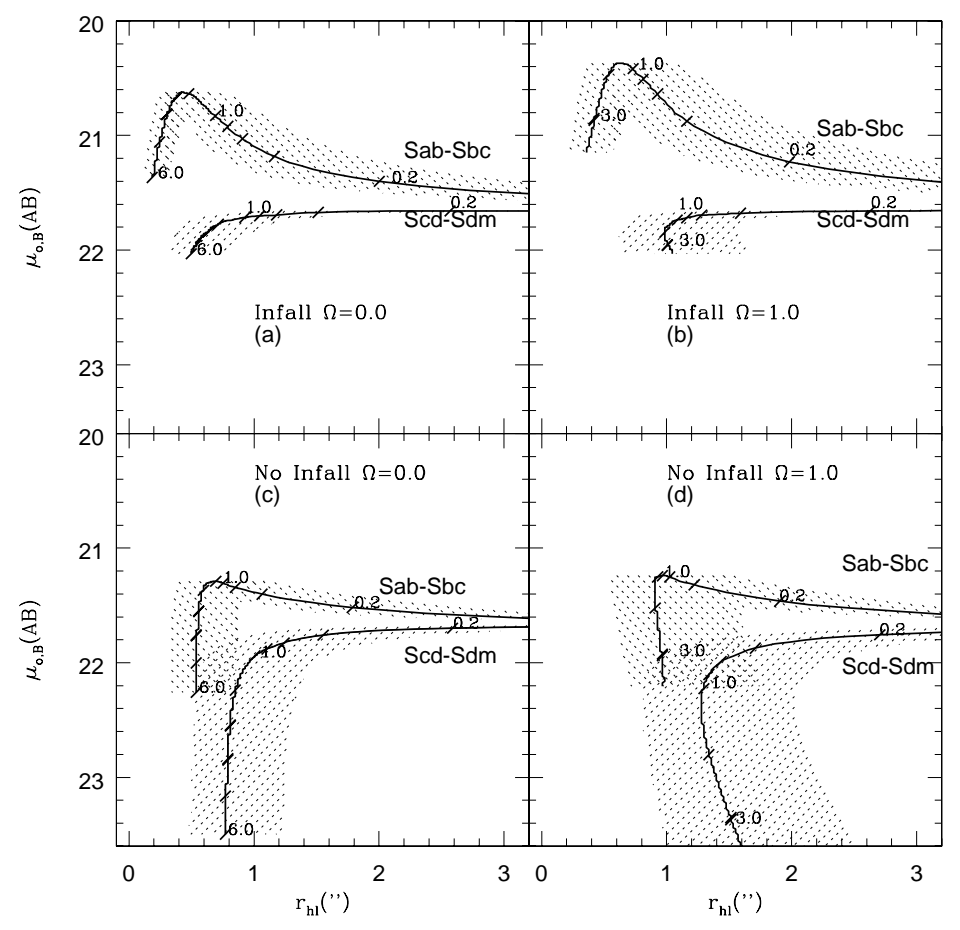

Fig. 1.- Disk central surface brightness $\mu_{o}$ versus half-light radius $r_{h l}$ for Sab-Sbc (top lines) and Scd-Sdm (bottom lines) galaxies. The redshift interval considered in the calculations extends from $z=0$ up to a redshift that corresponds to the time at which galaxies have been evolving 1 Gyr in the different models. Solid lines denote the values corresponding to a $M^{*}$ galaxy with the shaded areas covering one magnitude above and below this. Predictions of the "Infall" models are presented in panels (a) and (b) while the "No Infall" models are presented in panels (c) and (d). Predicted values in $\Omega=0.0$ and $\Omega=1.0$ cosmologies appear in the left panels and right panels respectively. Tick marks denote the locations at different redshifts in steps of 0.2 from $z=0$ to $z=1$ and in steps of 1 from $z=1$ to the highest redshift indicated for each model. 


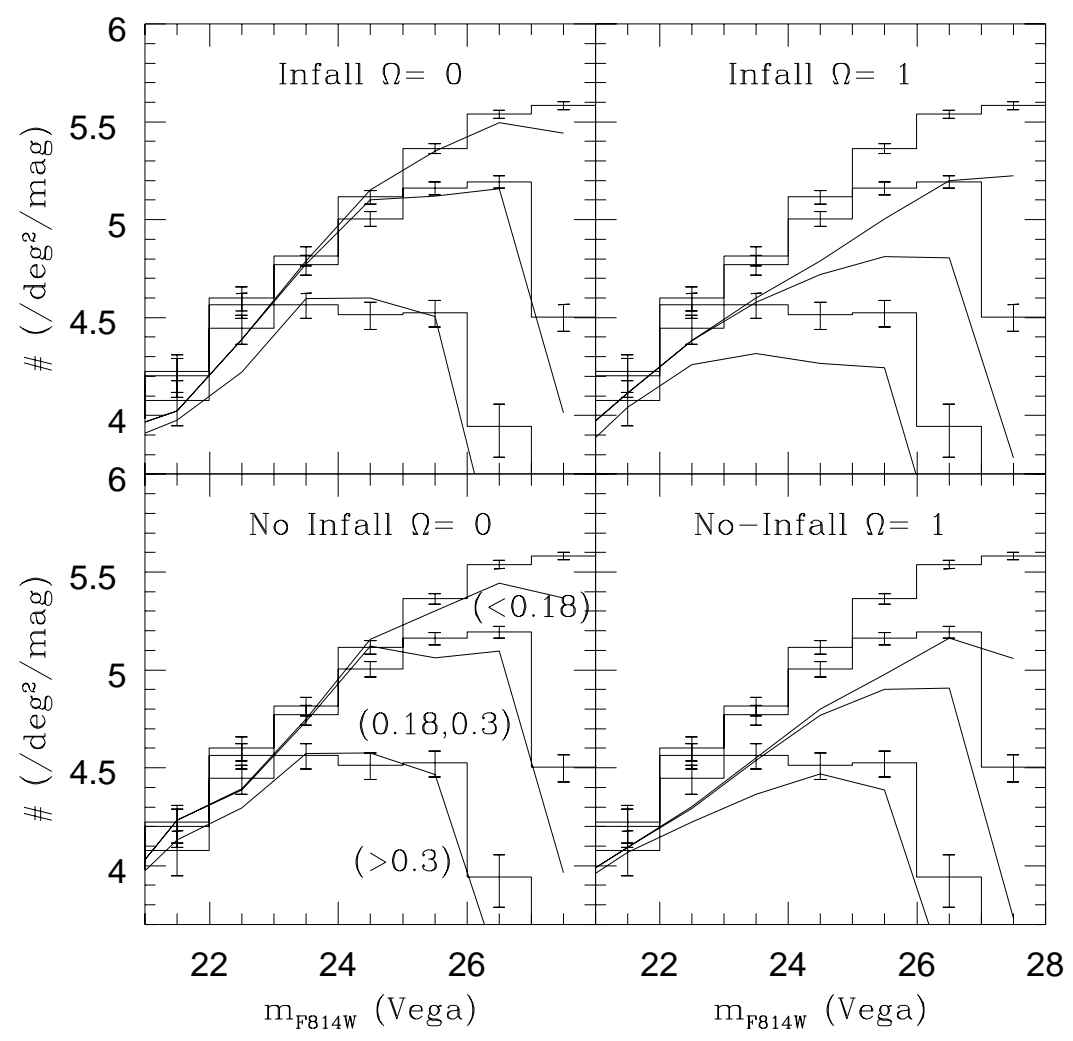

Fig. 2.- Comparison of the number counts recovered from the HDF with those recovered from the simulations. The observed number counts are broken up in terms of their measured half-light radii into three bins $r_{h l}<0.18$ arcsec, $0.18 \operatorname{arcsec}<r_{h l}<0.30 \operatorname{arcsec}$, and 0.30 $\operatorname{arcsec}<r_{h l}$. The error bars are the one-sigma poissonian values. 


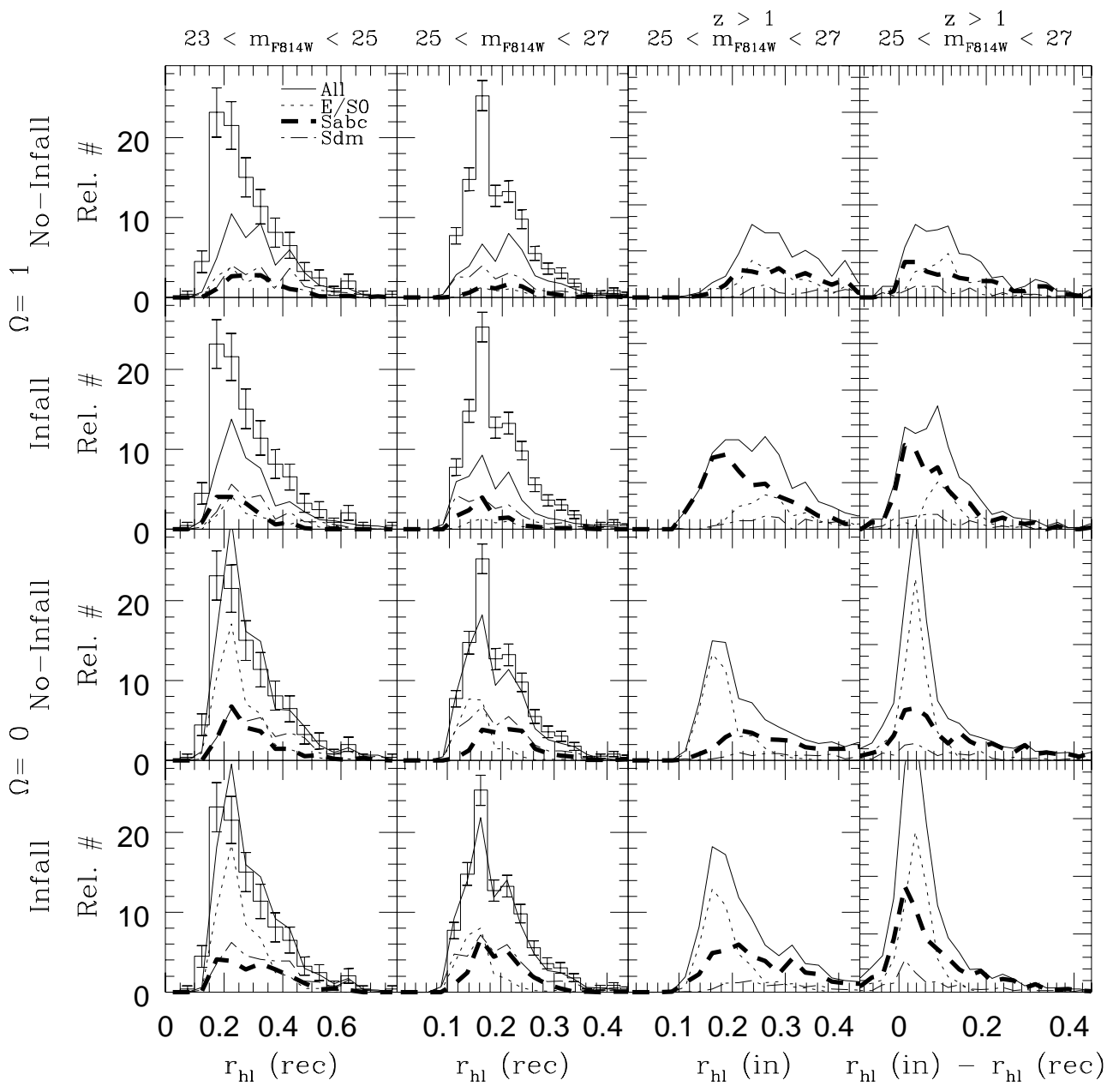

Fig. 3. - Comparison of the half-light radii distributions recovered from the HDF (histogram) with one-sigma error bars against those recovered $\left(\mathrm{r}_{h l}(\mathrm{rec})\right)$ from our simulations in the magnitude bins $\left(23<m_{F 814 W}\right.$ (Vega) $<25 ; 25<m_{F 814 W}$ (Vega $\left.)<27\right)$. The angular size distributions recovered from the simulations are decomposed into morphological types. The true half-light radii $\left(\mathrm{r}_{h l}(\mathrm{in})\right)$ distributions of higher redshift $(z>1)$ objects recovered in the magnitude bin $25<m_{F 814 W}$ (Vega) $<27$ are compared to the recovered distributions to illustrate both the errors in our procedure for determining the half-light radii and the fact that differences in half-light radii between the "Infall" and "No Infall" models are more apparent in the true half-light radii distributions than in the recovered distributions. 
Table 1. Model parameters ${ }^{a}$

\begin{tabular}{lrrrrrrrr}
\hline \hline$\Omega$ & Type & $\alpha$ & $M_{b_{J}}^{*}$ & $\phi_{0}^{*}$ & $\mathrm{~B} / \mathrm{T}^{b}$ & $\mu_{0}^{b_{J}}$ & SFR & T(Gyr) \\
\hline $0.0(1.0)$ & $\mathrm{E} / \mathrm{S} 0$ & -0.48 & -20.87 & 0.95 & 1 & - & $\tau_{1}^{c}\left(B_{1}^{d}\right)$ & $16.0(12.7)$ \\
$\ldots$ & Sab-Sbc $^{e}$ & -1.32 & -21.14 & 0.69 & 0.2 & 21.65 & $\tau_{f}=7.2 \mathrm{Gyr}(3.9 \mathrm{Gyr})$ & $18.0(12.7)$ \\
$\ldots$ & Sab-Sbc $^{e}$ & -1.32 & -21.14 & 0.69 & 0.13 & 21.65 & $\tau_{f}=7.2 \mathrm{Gyr}(3.9 \mathrm{Gyr})$ & $18.0(12.7)$ \\
$\ldots$ & Scd-Sdm $^{e}$ & -1.32 & -21.14 & 0.76 & 0 & 21.65 & $\tau_{f}=\infty \mathrm{Gyr}(\infty \mathrm{Gyr})$ & $18.0(12.7)$ \\
$\ldots$ & $\mathrm{Scd} \mathrm{Sdm}^{f}$ & -1.5 & -21.14 & 1.35 & 0 & 22.5 & $\tau_{f}=\infty \mathrm{Gyr}(\infty \mathrm{Gyr})$ & $18.0(12.7)$ \\
\hline
\end{tabular}

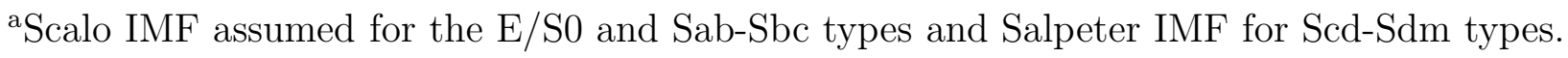

${ }^{\mathrm{b}}$ Bulge-to-total luminosity ratio in $b j$ band at $z=0$.

${ }^{c}$ Exponential SFR characterized by decay time $\tau_{1}=1 \mathrm{Gyr}$.

${ }^{\mathrm{d}} 1$ Gyr burst SFR.

${ }^{e} \mathrm{LF}$ truncated to include only galaxies with $M_{b j}<-18$.

${ }^{\mathrm{f}} \mathrm{LF}$ truncated to include only galaxies with $M_{b j}>-18$. 